CLINICAL STUDY

\title{
Metformin and placebo therapy both improve weight management and fasting insulin in obese insulin-resistant adolescents: a prospective, placebo-controlled, randomized study
}

\author{
Susanna Wiegand, Dagmar l'Allemand ${ }^{1}$, Hanna Hübel, Heiko Krude, Mareike Bürmann, Peter Martus ${ }^{2}$,
} Annette Grüters and Reinhard W Holl ${ }^{3}$

Department of Pediatric Endocrinology and Diabetology, Charité Children's Hospital, Universitätsmedizin Berlin, Augustenburger Platz 1, 13353 Berlin, Germany, ${ }^{1}$ Children's Hospital of Eastern Switzerland, 9000 St Gallen, Switzerland, ${ }^{2}$ Department of Epidemiology, Universitätsmedizin Berlin, 10117 Berlin, Germany and ${ }^{3}$ Department of Epidemiology, University of Ulm, 89081 Ulm, Germany

(Correspondence should be addressed to S Wiegand; Email: susanna.wiegand@charite.de)

\begin{abstract}
Objective: To study whether metformin reduces obesity, homeostasis model assessment for insulin resistance index (HOMA-IR), and the metabolic syndrome (MtS) in obese European adolescents in addition to previous unsuccessful lifestyle intervention.

Design and methods: After 6 months of multiprofessional lifestyle intervention, 70 out of 86 adolescents without improvement in body mass index (BMI) and HOMA-IR were randomized into either the placebo $(n=34)$ or the metformin group $(2 \times 500 \mathrm{mg} /$ day, $n=36)$ in addition to ongoing lifestyle intervention for another 6 months.

Results: Age was 13.8 years, BMI was $33.1 \mathrm{~kg} / \mathrm{m}^{2}, 65 \%$ were female, and $89 \%$ were Caucasians. During lifestyle intervention alone, BMI and HOMA-IR deteriorated significantly. In the subsequent medication period, HOMA-IR and fasting insulin improved similarly in the placebo and metformin groups (HOMA-IR decreased 73 vs $54 \%$ respectively in metformin versus placebo; $P=0.048$ ), but BMI remained unchanged. The insulin sensitivity index, however, only improved in the metformin group. High fasting insulin is correlated with a subsequent BMI increase irrespective of the medication. MtS remained unchanged.

Conclusions: Obese European adolescents' insulin sensitivity improved without weight change during placebo or metformin intervention in addition to lifestyle intervention. Most differences did not reach statistical significance, probably due to improved compliance with lifestyle intervention as a placebo effect. In addition, the metformin dose may be too low.
\end{abstract}

European Journal of Endocrinology 163 585-592

\section{Introduction}

The obesity epidemic challenges health care systems worldwide. Weight loss is known to improve cardiovascular risk factors (1). However, most therapeutic approaches do not induce sustained weight loss for all participants, even in well-established multiprofessional programs (2). The increase in childhood obesity due to environmental factors (i.e. increased consumption of processed food and physical inactivity) has led to a rising prevalence of the metabolic syndrome (MtS) and type 2 diabetes not only in minority groups but also in the general population (3). In obese adults with impaired glucose regulation, $5 \%$ per year convert to type 2 diabetes (4). Increased fasting insulin levels and insulin resistance (IR) during adolescence are major predictors of type 2 diabetes later in life (5). In the Diabetes Prevention Program in the United States (U. S. DPP), metformin was effective in preventing type 2 diabetes in adults with impaired glucose tolerance, but lifestyle intervention was even better. Also in children and adolescents, weight loss is known to decrease cardiovascular risk factors (6-9). Results for metformin therapy in overweight adolescents are conflicting. Individual effects of the intensive lifestyle intervention and of metformin have not been compared with the combination of both interventions being used simultaneously. In two small placebo-controlled pediatric trials, metformin was tested in obese non-diabetic adolescents $(10,11)$ : both the studies demonstrated significantly greater weight loss and reduced fasting insulin levels in the metformin group. More recently, a study with 28 obese adolescents reported a beneficial effect of metformin on body mass index (BMI), body composition, and fasting insulin but not on insulin sensitivity (12). Thus far, two studies considered the effect of metformin in addition to 
a lifestyle intervention program. The combination proved to be effective in reducing BMI and IR in a placebo-controlled study in obese Turkish adolescents with IR (13). In contrast, in a group of obese adolescents predominantly from ethnic groups at risk for type 2 diabetes, this 'add on' design showed no significant changes in metabolic parameters or BMI. Weight loss was significant only in girls with good metformin adherence and reported lifestyle changes (14).

A most recent meta-analysis (15) of these studies ascertained a moderate beneficial metformin effect on obesity outcomes among hyperinsulinemic children and adolescents but proposed to establish the role of metformin therapy in larger cohorts across different populations. In contrast to the conflicting results of controlled clinical studies, in clinical practice, metformin is used more and more in obese non-diabetic adolescents without licensed indication. For these reasons, we performed a double-blind, randomized controlled clinical trial in obese European adolescents at high risk for type 2 diabetes in addition to a previous unsuccessful lifestyle intervention.

\section{Methods}

\section{Lifestyle intervention}

Two hundred and seventy-eight obese children and adolescents (BMI $>97$ th percentile according to German references (16) were referred by general pediatricians to two pediatric obesity centers (Berlin, Germany and St Gallen, Switzerland). All the patients presented with obesity-related comorbidities, namely features of the MtS. Owing to additional factors, e.g. low education level (parental or personal), dysfunctional families, school problems, and an immigrant background, it was not feasible for these patients to participate in standardized obesity group programs. Instead, the patients and their families were invited to take part in a multiprofessional family-based individualized obesity treatment similar in both centers. They were asked to participate in a standardized program based on individual goal setting with compulsory participation of parents. Initially, a structured interview was performed to evaluate nine topics relating to weight reduction: five topics dealing with nutrition and eating behavior, two about physical activity, one dealing with media use (e.g. TV and computer), one each focussing on psychosocial and family problems. The interview was followed by basic information on healthy lifestyle appropriate for children and adolescents. This basic educational component was also adapted to these nine topics. Patients and families were subsequently assisted in one to three individually chosen tasks (goals) for a 1-2-month period (e.g. reducing TV watching from 4 to $2 \mathrm{~h}$ /day, walking to school $10 \mathrm{~min}$ instead of taking the school bus, and drinking tea without added sugar).
The lifestyle changes were recorded in simple 'token' systems adapted to adolescents with low education and language problems to support the maintenance of the goals. Multiprofessional reinforcement sessions took place every 4-8 weeks depending on the resources of the adolescents and their families. Regarding physical activity, the patients and families were to attend specialized sport classes for obese children and adolescents. Two sport classes per week ( $45 \mathrm{~min}$ each) was recommended, in addition to the regular sport classes at school. This lifestyle intervention was designed as a low-threshold service and was maintained throughout the entire duration of the trial, even after the medication was introduced.

\section{Study design}

Two hundred and seventy-eight obese adolescents at risk of developing type 2 diabetes according to American Diabetes Association (ADA) criteria (obesity, acanthosis nigricans, signs of the MtS, impaired fasting glucose, and positive family history of type 2 diabetes) (17), or with impaired glucose tolerance, applied for the individualized weight loss program and were screened. Eighty-six patients met the inclusion criteria (obese, 10-17 years, homeostasis model assessment for IR index (HOMA-IR)) $>3$ or $>95$ th percentile according to Allard et al. (18), non-diabetic, and normal liver and kidney function) and were enrolled in the study. Exclusion criteria included pre-existing diabetes, pregnancy, liver enzymes above 1.5 times the upper limit of normal or elevated creatinine $>1.5 \mathrm{mg} / \mathrm{dl}$, and severe chronic or mental illness. After ongoing participation in the weight loss program for at least 6 months from baseline (T1-T2), the 86 patients were reexamined. Fourteen patients reduced their body weight significantly $\left(\Delta \mathrm{BMI}>2 \mathrm{~kg} / \mathrm{m}^{2}\right)$ and/or no longer insulin resistant. Two patients dropped out for unknown reasons. The remaining 70 patients with unsuccessful lifestyle intervention (defined as reduction of obesity by $\Delta \mathrm{BMI}$ $<2 \mathrm{~kg} / \mathrm{m}^{2}$ and persistent IR expressed as HOMA-IR $>3$ or $>95$ th percentile according to Allard et al. (18)) were enrolled in the medication phase of the study (T2-T4). The sample patients were randomized for age, sex, and pubertal stage, and received either metformin $500 \mathrm{mg}$ twice/day or placebo for 6 months between 05/2006 and 12/2006 (administered in a double-blind way). Placebo or metformin tablets were dispensed by the local pharmacy, and unused pills were counted after 3 (T3) and 6 months (T4) to assess compliance. At 3 months of medication, a clinical and biochemical assessment of health status and side effects was conducted. After 6 months of medication, the patients were retested. The multiprofessional individualized lifestyle intervention continued for the whole study period ('Add on' design between T2 and T4). Informed consent was obtained from the study subjects and parents. The protocol for this randomized, double-blind, 


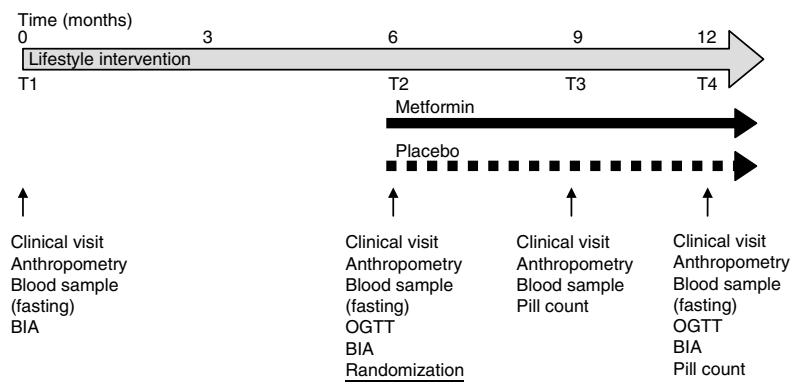

Figure 1 Time line for interventions and investigations. OGTT, oral glucose tolerance test; BIA, bioelectrical impedance analysis.

placebo-controlled clinical trial was approved by the local ethic committees (Charité Berlin, Germany; St Gallen Switzerland), monitored by the local centers for clinical studies, and registered at the European Study Council (Eudra-CT-Nr 2004-003816-47) (Fig. 1).

\section{Investigations}

During visits at T1, T2, and T4 (Fig. 1), family history for type 2 diabetes and MtS, ethnicity, anthropometric data, blood pressure, and pubertal status were recorded. Body weight and height were measured with calibrated instruments (Soehnle digital scale; Germany and wall mounted stadiometer; Keller, Germany) to the nearest $0.1 \mathrm{~kg}$ and $0.5 \mathrm{~cm}$ respectively. BMI was calculated (weight in kilograms divided by the square of the height in meters) using German reference data for the definition of overweight ( $>90 \mathrm{BMI}$ percentile and $\leq 97 \%$ and obesity (>97\%) (15). Blood pressure was measured in a supine position after $5 \mathrm{~min}$ rest to the nearest $2 \mathrm{mmHg}$ using a Dinamap model, and results were interpreted using percentiles for age and sex (19). Pubertal status was assessed according to Tanner's criteria $(20,21)$.

After a 12-h overnight fast, these participants underwent an oral glucose tolerance test (OGTT) at $0800 \mathrm{~h}$. Baseline samples were obtained for measurement of fasting glucose, fasting insulin, lipids (cholesterol (chol.), high-density lipoprotein cholesterol (HDL-chol.), low-density lipoprotein cholesterol (LDL-chol.), and triglycerides), aspartate aminotransferase, alanine aminotransferase, and creatinine. Glucose was administered orally $(1.75 \mathrm{~g} / \mathrm{kg}$, up to a maximum of $75 \mathrm{~g}$ glucose). Blood samples were drawn after 30, 60, 90, and $120 \mathrm{~min}$. Glucose and insulin concentrations were determined and categorized using World Health Organization criteria (22). All the participants followed a diet consisting of at least $250 \mathrm{~g}$ carbohydrates/day for 3 days before the study.

\section{Biochemical analyses}

Glucose was measured by the glucose oxidase method with venous whole blood being immediately deproteinized with perchloric acid. Insulin was measured with the AutoDELFIA Insulin assay (Perkin Elmer, Turku, Finland). The cross-reactivity with C-peptide was $<0.18 \%$, and with proinsulin was $<1 \%$. The intra- and inter-assay coefficients of variation for insulin were 3.4 and $2.8 \%$ respectively. The lipid levels were determined by an enzymatic colorimetric test (analyzer 704/717 Roche-Hitachi). To measure liver and kidney functions, the standardized assay procedures at the Children's Hospitals were used.

Two approaches were used to characterize insulin sensitivity: the HOMA-IR (23), an inverse surrogate parameter of insulin sensitivity validated in obese and non-obese children and adults $(17,24,25)$, was estimated using the formula:

HOMA-IR $=\frac{\text { Fasting insulin }(\mathrm{mIU} / \mathrm{l}) \times \text { fasting glucose }(\mathrm{mmol} / \mathrm{l})}{22.5}$.

Insulin sensitivity was determined by the insulin sensitivity index (ISI) from Matsuda \& De Fronzo (26).

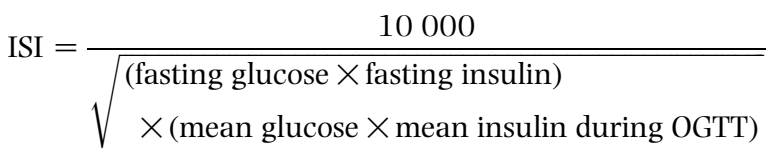

where glucose is measured in mmol/l and insulin in $\mathrm{mIU} / \mathrm{l}$.

For HOMA-IR, values above the 95th percentile for age and gender were used as cutoff (18). For ISI, normal values for children and adolescents are not available.

\section{Statistical methods}

The primary hypothesis of this study was the improvement of IR measured by HOMA-IR at T4 in comparison to T1 and T2. Secondary endpoints were anthropometric measurements (BMI and waist-to-hip ratio), cardiovascular risk parameters (systolic and diastolic blood pressure), lipid profile (total-, LDL-, HDL-chol., and triglycerides), and other metabolic parameters (glucose tolerance and fasting insulin). In addition, covariates included age, gender, and pubertal stage. Since a clinically significant effect was defined as a decrease in HOMA-IR by -1 , two groups of 37 patients had to be included in the study to achieve a power of 0.9 with $\alpha$ value of 0.05 . Descriptive analysis included means, s.D., medians, quartiles, and ranges for each variable separately in both the study arms. For each of the parameters, normal distribution was examined, and, if necessary, a log transformation was applied. For both primary and secondary endpoints, the primary analysis was the analysis of covariance with baseline as the continuous covariate and each study arm as the factor. Additionally, the influence of potential predictors for change in HOMA-IR was analyzed using analysis of covariance. The $\alpha$-level was set at 0.05 (two-sided) for all statistical tests, only the primary analysis was strictly confirmatory. The analyses were done using SAS release 9.1 (SAS Institute, Cary, NC, USA) and SPSS 15.0 (SPSS Inco, Chicago, IL, USA). 


\section{Results}

Eighty-six obese insulin-resistant adolescents consented to participate in the study (Fig. 2, mean age 13.8 \pm 2.1 years; mean BMI $33.1 \pm 4.6 \mathrm{~kg} / \mathrm{m}^{2}$ ). In comparison to patients who were not enrolled, the study cohort was predominantly female, older, and further advanced in puberty. Fasting insulin as well as systolic blood pressure was significantly higher in the study cohort (Table 1).

After 6 months of lifestyle intervention, 70 patients who did not significantly reduce BMI or IR were randomized to either metformin $(n=36)$ or placebo $(n=34)$ group. The groups did not differ significantly by gender (female 72 vs $62 \% ; P=0.35$ ), age ( 15.1 vs 15.0 years; $P=0.98$ ), ethnicity (Caucasian 87.3 vs $91.2 \%$; $P=0.11$ ), pubertal stage (Tanner $>$ III 87.1 vs $90.5 \%$; $P=0.71)$, or BMI (34.3 vs $\left.35.5 \mathrm{~kg} / \mathrm{m}^{2} ; P=0.35\right)$.

\section{Adherence to lifestyle intervention prior to and during medication}

For the study population, the adherence to the lifestyle intervention program was significantly improved during the total medication period (between T2 and T4; Fig. 1) in comparison to the lifestyle intervention alone (between T1 and T2). Appointments for clinical visits and educational sessions were kept in $62 \%$ of the cases in the first 6 months without medication. The adherence rate increased to $88 \%(P=0.001$; metformin group $)$ and $92 \%(P=0.001$; placebo group $)$ after randomization. While the clinical setting of lifestyle intervention and the intensity of care did not change throughout the study, individual goals were diverse before and after randomization, with the majority of participants choosing to reduce their soft drink consumption during the medication period. During the lifestyle intervention alone, $32 \%$ reported that they used water to quench their thirst, in contrast to $74 \%$ during medication plus

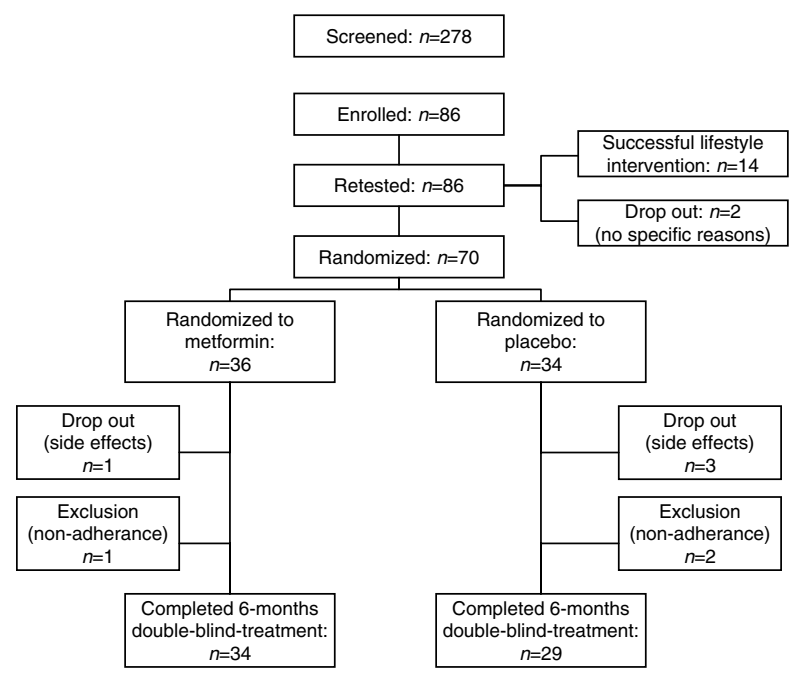

Figure 2 Enrolment of the patients and completion of the study. lifestyle intervention $(P<0.001)$. Concerning energy expenditure, the adherence to regular physical activity also increased significantly between T2 and T4 (medication plus lifestyle intervention): significantly, more adolescents $(61 \%$ in the metformin group and $70 \%$ in the placebo group) reported performing at least one sport session of 45-90 $\mathrm{min} /$ week in addition to school physical education, compared with $23 \%(P<0.001)$ and $28 \%(P<0.001)$ respectively between $\mathrm{T} 1$ and $\mathrm{T} 2$ (exclusive lifestyle intervention). Self-reported media time of more than $3 \mathrm{~h} /$ day was present in $67 \%$ during lifestyle alone (between T1 and T2) and in 43\% during medication plus lifestyle intervention (between $\mathrm{T} 2$ and T4; $P=0.032$ ).

\section{Side effects, adherence to medication, and drop out}

In general, metformin and placebo were well tolerated. Gastrointestinal symptoms were reported in $20 \%$ of the participants (metformin $n=5$; placebo $n=9$ ). Specific treatment was not necessary. These side effects caused $6 \%$ of the participants to drop out of the study (metformin $n=1$; placebo $n=3$; Fig. 2). Additionally, $10 \%$ of the participants (metformin $n=3$; placebo $n=4$ ) reported unspecific side effects such as weakness or fatigue for a short time, with spontaneous remission.

Three participants were excluded from the study due to insufficient adherence based on pill counts (intake $<70 \%$; metformin $n=1$; placebo $n=2$ ). Therefore, the total dropout rate after randomization was $10 \%(n=7)$, five in the placebo group and two in the metformin group (Fig. 2).

\section{Anthropometric and metabolic parameters}

Between $\mathrm{T} 1$ and $\mathrm{T} 2$, the participants gained weight $\left(n=70 ; \quad\right.$ BMI $33.1 \pm 4.7$ to $34.9 \pm 5.4 \mathrm{~kg} / \mathrm{m}^{2}$; $P<0.001)$, and fasting blood glucose $(n=70 ; 87.7$ \pm 6.7 to $94.7 \pm 10.6 \mathrm{mg} / \mathrm{dl} ; \quad P<0.001)$ as well as HOMA-IR $(n=70 ; 5.0 \pm 2.7$ to $5.9 \pm 3.1 ; P<0.001)$ increased significantly. The effects of metformin and the placebo in addition to the ongoing lifestyle intervention are shown in Table 2. In both the groups, the primary outcome parameter, HOMA-IR, increased between T1 and T2 (exclusive lifestyle intervention), and significantly improved between $\mathrm{T} 2$ and $\mathrm{T} 4$ (added medication) (Fig. 2).

Though a reduction in BMI could be observed in 53\% (metformin) and 36\% (placebo) of the patients, changes between T2 and T4 and group differences were not significant (intergroup $P=0.054$; Table 2). This was also the case for waist-to-hip ratio and body composition. (Bioimpedance analysis, data not shown). Concerning the metabolic parameters (Table 2), fasting insulin decreased significantly, both with metformin (23.3 to $18.8 \mu \mathrm{U} / \mathrm{ml}$ ) and with the placebo $(27.7$ to $22.2 \mu \mathrm{U} / \mathrm{ml}$ ). HOMA-IR also dropped in both the groups significantly (metformin 5.35-4.8 and placebo 
Table 1 Baseline characteristics of the study cohort.

\begin{tabular}{|c|c|c|c|}
\hline Parameters & $\begin{array}{l}\text { Patients enrolled } \\
\text { in the metformin } \\
\text { study at T1 } n=86\end{array}$ & $\begin{array}{l}\text { Patients at risk } \\
\text { of diabetes not } \\
\text { enrolled } n=192\end{array}$ & $P$ value \\
\hline \multicolumn{4}{|l|}{ Gender } \\
\hline Male & $30(34.9 \%)$ & $98(51 \%)$ & 0.012 \\
\hline Female & $56(65.1 \%)$ & $94(49 \%)$ & \\
\hline Age (years) & $13.77 \pm 2.13$ & $12.88 \pm 3.17$ & 0.007 \\
\hline \multicolumn{4}{|l|}{ Ethnicity } \\
\hline Caucasian & $80(93 \%)$ & $173(90.1 \%)$ & 0.585 \\
\hline Others & $6(7 \%)$ & $18(9.9 \%)$ & \\
\hline \multicolumn{4}{|l|}{ Pubertal stage (Tanner) } \\
\hline Prepubertal (I) & $6(7.1 \%)$ & $43(22.4 \%)$ & \\
\hline Early puberty (II and III) & $23(27.4 \%)$ & 69 (35.9\%) & \\
\hline Late puberty (IV and V) & $55(65.5 \%)$ & $80(41.7 \%)$ & \\
\hline BMI $\left(\mathrm{kg} / \mathrm{m}^{2}\right)$ & $33.06 \pm 4.64$ & $32.93 \pm 6.56$ & 0.851 \\
\hline BMI-SDS & $2.72 \pm 0.49$ & $2.79 \pm 0.60$ & 0.389 \\
\hline HOMA-IR & $4.9(3.9-5.98)$ & $4.58(3.05-7.10)$ & 0.139 \\
\hline Fasting insulin $(\mu \mathrm{U} / \mathrm{ml})$ & $23.8(19.1-28.5)$ & $21.10(13.78-31.83)$ & 0.015 \\
\hline Fasting blood glucose $(\mathrm{mg} / \mathrm{dll})$ & $84.28 \pm 15.58$ & $89.40 \pm 8.41$ & 0.06 \\
\hline Systolic blood pressure (mmHg) & $128.68 \pm 10.59$ & $124.42 \pm 13.87$ & 0.008 \\
\hline Diastolic blood pressure $(\mathrm{mmHg})$ & $67.67 \pm 8.58$ & $69.39 \pm 9.55$ & 0.175 \\
\hline
\end{tabular}

Data are shown as means \pm s.D., medians (1-3 quartile), or number $n(\%)$ in percent of total. BMI, body mass index; HOMA-IR, homeostasis model assessment of insulin resistance, refer to Methods. To convert values for insulin to picomoles per liter, multiply by 7.17 . To convert values for glucose to millimoles per liter, multiply by 0.05551 . Significant differences of values $P<0.05$ are indicated in bold.

6.65-5.6). ISI improved significantly only in the metformin group $(0.70-1.1 ; P=0.008)$. Overall, IR indices (HOMA-IR or ISI) improved in $73 \%$ of the metformin-treated patients and in $54 \%$ of the placebo group (intergroup $P=0.048$ ).

Cardiovascular risk parameters remained unchanged in the metformin and the placebo groups between T2 and T4 (Table 2).

Regarding the changes of adiposity over time and IR indices, HOMA-IR and fasting insulin at T1 were significantly correlated to BMI at T4 $(r=0.44$, $P<0.001$ and $r=0.42, P=0.001$ respectively; corrected for age and pubertal stage) but not to BMI at T2. No other significant correlations were observed.

\section{Discussion}

Subjects included in the present study represent a high risk group of extremely obese, late pubertal, and predominantly female European adolescents with significantly higher systolic blood pressure and fasting insulin levels than the patients who were not enrolled in the outpatient obesity clinic. Moreover, it has to be borne in mind that the study was conducted in a population with a physiologically high IR due to pubertal development (18), which might be reflected by the slight increase in fasting glucose over the whole study period in both the study arms. During individualized multiprofessional lifestyle intervention alone, BMI and IR deteriorated significantly in the study cohort. During both the placebo and metformin therapy in addition to lifestyle intervention, however, insulin sensitivity improved without weight change.
The significant improvements in HOMA-IR and fasting insulin as well as in better weight regulation during medication both in the study and the placebo groups suggest a strong indirect placebo effect. Patients could have been highly motivated through enrolment, as they became significantly more engaged in the obesity program and were ready to undertake individual changes in nutritional habits and physical activity. Especially, the reduction in soft drink consumption as an individual goal potentially influenced metabolic parameters due to the reduced sucrose, glucose, and fructose intake (27). This might also modulate central appetite regulation (28). Self-reported lifestyle changes only reflect the subjective appraisals of patients and families. This limits the force of expression concerning the significant differences between lifestyle alone and medication plus lifestyle, independent of metformin or placebo.

While most differences between metformin and placebo did not reach statistical significance, significant improvement of insulin sensitivity was observed only in the metformin group. As an indicator, ISI particularly reflects the peripheral insulin sensitivity, which is primarily deteriorated in obese children and adolescents. Therefore, this points to a notable metformin effect in this field. In relation to adult studies, the values for ISI are relatively very low, suggesting severe IR in this group. As most patients are in the puberty stage, this is an additional condition with physiological IR. This supports the well-known efficacy of metformin therapy on glucose metabolism and its effects in counteracting IR, but the dose of $1000 \mathrm{mg}$ metformin/day was probably too low for the body mass of these adolescents to reveal effects in addition to 


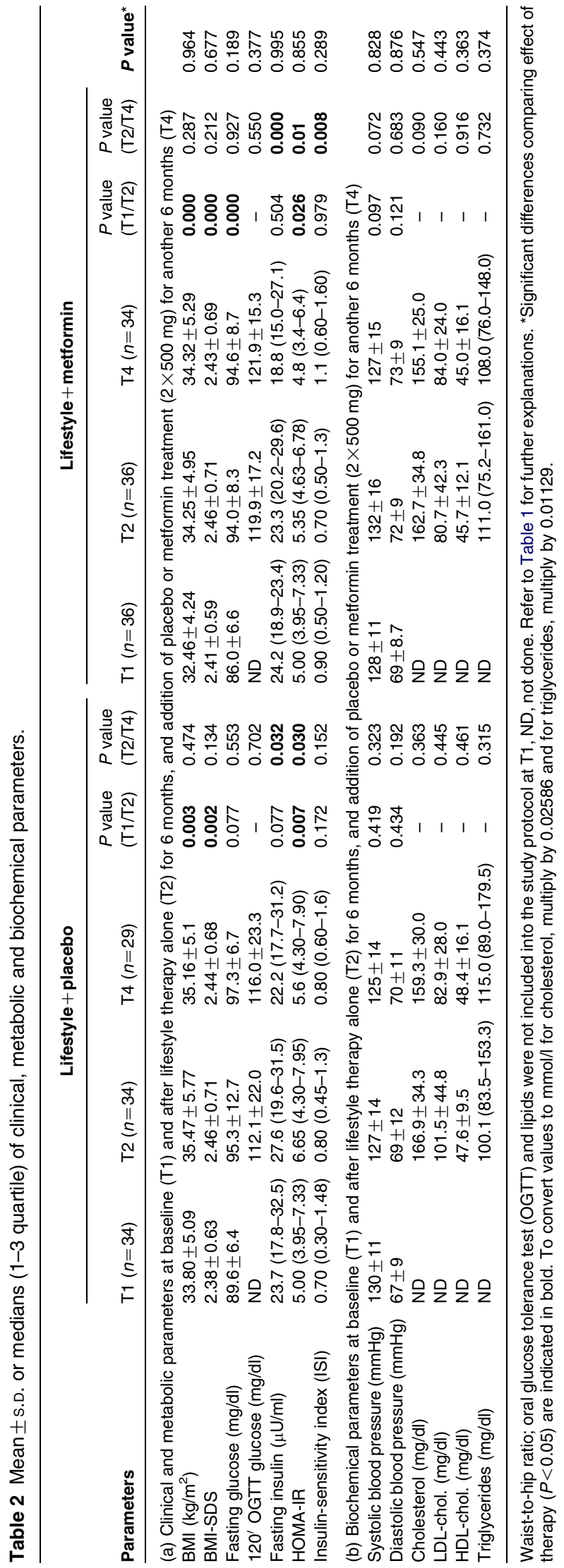

an efficacious lifestyle intervention. This dose was strongly recommended by the ethics committees because of the pediatric study population, and a step-wise increase of the dose was not permitted. This is partially supported by further studies with metformin in pre-diabetic or insulin-resistant obese adolescents. Three previous prospective randomized studies performed in child cohorts with $<30$ patients each (10-12) suggest a beneficial effect of metformin, however with low significance and without additional lifestyle modification.

Our study supports the findings by Love-Osborne et al. (14) with 85 obese, insulin-resistant adolescents. This study did not show a significant improvement in weight loss in subjects receiving metformin in addition to lifestyle modification, except for females reporting a decrease in food portion size. This study population predominantly included adolescents from ethnic groups at risk for the development of type 2 diabetes and with extreme obesity (mean BMI $39 \mathrm{~kg} / \mathrm{m}^{2}$ ). In the presented clinical trial, the majority of patients were also extremely obese (mean BMI $34 \mathrm{~kg} / \mathrm{m}^{2}$ ) but were Caucasian. Therefore, the results complement one another (29). In contrast to the reported data, Atabek et al. (13) describe a significant benefit of $1000 \mathrm{mg}$ metformin/day in a randomized, placebo-controlled, double-blind study in 90 obese Turkish adolescents regarding BMI, fasting insulin, and HOMA-IR. This study population was less obese (mean BMI $28.5 \mathrm{~kg} / \mathrm{m}^{2}$ ). Burgert et al. (30) tested the metabolic and cardiovascular effects of $1500 \mathrm{mg}$ metformin in extremely obese non-diabetic adolescents (randomized, placebo-controlled and double-blind) in a 4-month trial. This short-term use of metformin had a beneficial effect on BMI and the autonomous nerve system (heart), but seemed to benefit insulin sensitivity only slightly. The correlation between initial fasting insulin levels and the weight outcome stresses the importance to treat hyperinsulinemia as a prerequisite for efficacious weight management. Insulin sensitization might be achieved by qualitative improvement of diet and activity, as in the present study, and/or by metformin in those unable to adhere to lifestyle modifications.

The lack of significant difference in HOMA-IR and BMI in the metformin-treated group versus placebo group does not support the use of metformin in the fixed dose of $1000 \mathrm{mg}$ in childhood to improve IR in addition to a multiprofessional obesity program, and thus further studies are necessary (15). The effectiveness of metformin, however, does not outrank that of behavioral interventions in reducing BMI. A meta-analysis showed that the latter results in a BMI reduction of $-3.04 \mathrm{~kg} / \mathrm{m}^{2}(-3.14$ to -2.94$)$ at 6 months, an effect that could still be observed at the 12-month follow-up (Oude Luttikhuis et al. (2009) (2)). Also when compared with other drugs licensed for the treatment of obesity, metformin only brings about a relatively moderate weight reduction (15). 
The present study, however, reveals a beneficial 'sideeffect'; there is a strong placebo effect being endorsed by the higher prevalence of side effects in the placebo group than in the metformin group. This placebo effect shows how important the belief in therapy effects and selfefficacy may be (31), as it strengthens the motivation for lifestyle changes in obese adolescents and enhances adhesion to a healthy lifestyle.

\section{Declaration of interest}

The authors declare that there is no conflict of interest that could be perceived as prejudicing the impartiality of the research reported.

\section{Funding}

The study was supported in part by BMBF Research grant 01 GS 0825 and by MERCK SANTE S.A.S, Lyon, France (10'000,- Euro); metformin, purchased at Hexal AG, Holzkirchen, Germany. The study protocol is registered at the European Study Council (Eudra-CT-Nr 2004-003816-47).

\section{Acknowledgements}

The authors wish to thank all the patients, families, investigators, and staff who participated in the study.

\section{References}

1 Reinehr T, de Sousa G, Toschke AM \& Andler W. Long-term follow-up of cardiovascular disease risk factors in children after an obesity intervention. American Journal of Clinical Nutrition 2006 84 490-496.

2 Oude Luttikhuis H, Baur L, Jansen H, Shrewsbury VA, O'Malley C, Stolk RP \& Summerbell CD. Interventions for treating obesity in children. Cochrane Database of Systematic Reviews 200921 CD001872. (doi:10.1002/14651858.CD001872.pub2)

3 Wiegand S, Maikowski U, Blankenstein O, Biebermann $\mathrm{H}$, Tarnow P \& Grüters A. Type 2 diabetes and impaired glucose tolerance in European children and adolescents with obesity - a problem that is no longer restricted to minority groups. European Journal of Endocrinology 2004151 199-206. (doi:10.1530/eje.0. 1510199)

4 Knowler WC, Barrett-Connor E, Fowler SE, Hamman RF, Lachin JM, Walker EA, Nathan DM \& Diabetes Prevention Program Research Group. Reduction in the incidence of type 2 diabetes with lifestyle intervention or metformin. New England Journal of Medicine 2002346 393-403. (doi:10.1056/NEJMoa 012512)

5 Nguyen QM, Srinivasan SR, Xu JH, Chen W \& Berenson GS. Changes in risk variables of metabolic syndrome since childhood in pre-diabetic and type 2 diabetic subjects: the Bogalusa Heart Study. Diabetes Care 200831 2044-2049. (doi:10.2337/dc08-0898)

6 Ferguson MA, Gutin B, Le N-A, Karp W, Litaker M, Humphries M, Okuyama T \& Owens S. Effects of exercise training and its cessation on components of the insulin resistance syndrome in obese children. International Journal of Obesity 199922 889-895. (doi:10.1038/sj.ijo.0800968)

7 Kang HS, Gutin B, Barbeau P, Owens S, Lemmon CR, Allison J, Litaker MS \& Le NA. Physical training improves insulin resistance syndrome markers in obese adolescents. Medicine and Science in Sports and Exercise 200234 1920-1927. (doi:10.1097/ 00005768-200212000-00010)

8 Grey M, Berry D, Davidson M, Galasso P, Gustafson E \& Melkus G. Preliminary testing of a program to prevent type 2 diabetes among high-risk youth. Journal of School Health 200474 10-15. (doi:10. 1111/j.1746-1561.2004.tb06595.x)
9 Roemmich JN, Liu EY, Rogol AD, Epstein LH \& Quattrin T. Diminished insulin resistance with weight loss in severely overweight youth. Metabolic Syndrome and Related Disorders 2004 2 160-168. (doi:10.1089/met.2004.2.160)

10 Kay JP, Alemzadeh R, Langley G, D'Angelo L, Smith P \& Holshouser S. Beneficial effects of metformin in normoglycemic morbidly obese adolescents. Metabolism 200150 1457-1461. (doi:10.1053/meta.2001.28078)

11 Freemark M \& Bursey D. The effects of metformin on body mass index and glucose tolerance in obese adolescents with fasting hyperinsulinemia and a family history of type 2 diabetes. Pediatrics 2001107 e55. (doi:10.1542/peds.107.4.e55)

12 Srinivasan S, Ambler GR, Baur LA, Garnett SP, Tepsa M, Yap F, Ward GM \& Cowell CT. Randomized, controlled trial of metformin for obesity and insulin resistance in children and adolescents: improvement in body composition and fasting insulin. Journal of Clinical Endocrinology and Metabolism 200691 2074-2080. (doi:10.1210/jc.2006-0241)

13 Atabek ME \& Pirgon O. Use of metformin in obese adolescents with hyperinsulinemia: a 6-month, randomized, double-blind, placebo-controlled clinical trial. Journal of Pediatric Endocrinology and Metabolism 200821 339-348.

14 Love-Osborne K, Sheeder J \& Zeitler P. Addition of metformin to a lifestyle modification program in adolescents with insulin resistance. Journal of Pediatrics 2008152 817-822. (doi:10. 1016/j.jpeds.2008.01.018)

15 Park MH, Kinra S, Ward KJ, White B \& Viner RM. Metformin for obesity in children and adolescents: a systematic review. Diabetes Care 200932 1743-1745. (doi:10.2337/dc09-0258)

16 Kromeyer-Hausschild K, Wabitsch M \& Kunze D. Perzentile für den Body-mass-Index für das Kindes-und Jugendalter unter Heranziehung verschiedener deutscher Stichproben. Monatsschr Kinderheilkd 2001149 807-818. (doi:10.1007) s001120170107)

17 Rhodes ET, Finkelstein JA, Marshall R, Allen C, Gillman MW \& Ludwig DS. Screening for type 2 diabetes mellitus in children and adolescents: attitudes, barriers, and practices among pediatric clinicians. Ambulatory Pediatrics 20066 110-114. (doi:10.1016/ j.ambp.2005.10.005)

18 Allard P, Delvin EE, Paradis G, Hanley JA, O’Loughlin J, Lavallee C, Levy E \& Lambert M. Distribution of fasting plasma insulin, free fatty acids, and glucose concentrations and of homeostasis model assessment of insulin resistance in a representative sample of Quebec children and adolescents. Clinical Chemistry 200349 644-649. (doi:10.1373/49.4.644)

19 Report of the Second Task Force on Blood Pressure Control in children. Task force on blood pressure control in children. Pediatrics 198779 1-25.

20 Marshall T. Variations in patterns of pubertal changes in girls. Archives of Disease in Childhood 197944 291-303. (doi:10.1136/ adc.44.235.291)

21 Marshall T. Variations in patterns of pubertal changes in boys. Archives of Disease in Childhood 197945 13-23. (doi:10.1136/adc. 45.239.13)

22 Alberti Z. New diagnostic criteria and classification of diabetesagain? Diabetic Medicine 199815 535-536. (doi:10.1002/(SICI) 1096-9136(199807)15:7<535::AID-DIA670> 3.0.CO;2-Q)

23 Matthews DR, Hosker JP, Rudenski AS, Naylor BA, Treacher DF \& Turner RC. Homeostasis model assessment: insulin resistance and beta-cell function from fasting plasma glucose and insulin concentrations in man. Diabetologia 198528 412-419. (doi:10. 1007/BF00280883)

24 Sinha R, Fisch G, Teague B, Tamborlane WV, Banyas B, Allen K, Savoye M, Rieger V, Taksali S, Barbetta G, Sherwin RS \& Caprio S. Prevalence of impaired glucose tolerance among children and adolescents with marked obesity. New England Journal of Medicine $2002 \quad 346 \quad 802-810$. (doi:10.1056/ NEJMoa012578)

25 Bonora E, Targher G, Alberiche M, Bonadonna RC, Saggiani F, Zenere MB, Monauni T \& Muggeo M. Homeostasis model assessment closely mirrors the glucose clamp technique in the 
assessment of insulin sensitivity: studies in subjects with various degrees of glucose tolerance and insulin sensitivity. Diabetes Care 200023 57-63. (doi:10.2337/diacare.23.1.57)

26 Matsuda M \& DeFronzo RA. Insulin sensitivity indices obtained from oral glucose tolerance testing: comparison with the euglycemic insulin clamp. Diabetes Care 199922 1462-1470. (doi:10.2337/diacare.22.9.1462)

27 Stanhope KL \& Havel PJ. Endocrine and metabolic effects of consuming beverages sweetened with fructose, glucose, sucrose, or high-fructose corn syrup. American Journal of Clinical Nutrition $2008 \mathbf{8 8}$ 1733S-1737S. (doi:10.3945/ajcn.2008. 25825D)

28 Melanson KJ, Angelopoulos TJ, Nguyen V, Zukley L, Lowndes J \& Rippe JM. High-fructose corn syrup, energy intake, and appetite regulation. American Journal of Clinical Nutrition 2008 88 1738S-1744S. (doi:10.3945/ajcn.2008.25825E)
29 Kasa-Vubu JZ. Metformin as a weight-loss tool in "at-risk" obese adolescents: a magic bullet? (Comment) Journal of Pediatrics 2008 152 750-752. (doi:10.1016/j.jpeds.2008.03.006)

30 Burgert TS, Duran EJ, Goldberg-Gell R, Dziura J, Yeckel CW, Katz S, Tamborlane WV \& Caprio S. Short-term metabolic and cardiovascular effects of metformin in markedly obese adolescents with normal glucose tolerance. Pediatric Diabetes 20089 567-576. (doi:10.1111/j.1399-5448.2008.00434.x)

31 Lippke S, Wiedemann AU, Ziegelmann JP, Reuter T \& Schwarzer R. Self-efficacy moderates the mediation of intentions into behavior via plans. American Journal of Health Behavior 200933 521-529.

Received 4 July 2010

Accepted 16 July 2010 